\title{
Comparison of Antinuclear Autoantibodies Expression Between COPD and Lung Cancer Patients
}

\author{
Ying Zhang ${ }^{1}$, Zengsheng Chen ${ }^{2}$, Xiaomin Liang ${ }^{3}$ and Shuangmei Liu ${ }^{1 *}$ \\ ${ }^{1}$ Department of Medicine, Municipal Hospital Group, China \\ ${ }^{2}$ Department of Clinical Laboratory, Municipal Hospital Group, China \\ ${ }^{3}$ Department of Thoracic Surgery, Municipal Hospital Group, China
}

*Corresponding author: Shuangmei Liu, Department of Medicine, Municipal Hospital Group, Qingdao, China.

To Cite This Article: Ying Zhang, Zengsheng Chen, Xiaomin Liang, Shuangmei Liu. Comparison of Antinuclear Autoantibodies Expression Between COPD and Lung Cancer Patients. Am J Biomed Sci \& Res. 2021 - 12(5). AJBSR.MS.ID.001797. DOI: 10.34297/AJBSR.2021.12.001797.

Received: 眥 March 08, 2021; Published: 㘹 May 04, 2021

\begin{abstract}
Objectives: Circulating antinuclear autoantibodies (ANAs) have been detected in lung cancer patients and chronic obstructive pulmonary disease (COPD) patients as well. The specific differences and significance of ANAs in COPD patients and lung cancer patients remain unclear.

Methods: We investigated the expression of circulating ANAs and their various known antibody subtypes in lung cancer patients, COPD patients and healthy controls, and compared the differences between each group, also the differences between patients over 70 and under 70 of age in each group and the differences between different genders. ANAs were tested by indirect immunofluorescence (IFT) and western blot analysis, and casecontrol study was used to investigate the differences between groups of 90 cases of lung cancer; 68 cases of COPD and 30 cases controls who were gender and age-matched.

Results: The results of the research showed that the positive rate of ANAs in lung cancer patients was significantly increased compared with COPD patients $(\mathrm{p}=0.004)$ and healthy controls $(\mathrm{P}=0.00)$. Statistical analysis did not reveal any correlations between the patients' age and the ANA positive results either in lung cancer group or in COPD group. The differences between different genders were not statistically significant; too. The most common autoantibody subtype was anti-Ro-52.
\end{abstract}

Conclusions: The positive rate of circulating ANAs was significantly higher in lung cancer patients than in COPD patients.

Keywords: Chronic Obstructive Pulmonary Disease; Lung Cancer; Antinuclear Autoantibodies; Indirect Immunofluorescence; Western Blot Analysis; Case-Control Study

\section{Introduction}

Antinuclear antibodies (ANAs) are commonly used as an initial test to screen for systemic autoimmune diseases, and the activity of autoantibody had been verified in the sera of cancer patients [1,2]. Emerging evidence suggests that ANAs are connected with COPD and low BMI, but not with smoking and forced expiratory volume in 1s [3]. Autoimmune are not rare among patients with cancer [1], previous study has shown that autoimmunity plays an important role in the pathogenesis of COPD [4]. Many epidemiological studies have agreed that the presence of COPD is associated with the development of lung cancer, but little is known about its mechanism
[5]. How to recognize the significance of ANAs elevation in clinical work is an important part of disease diagnosis and differential diagnosis.

We conducted a case-control study to further clarify the characteristics of ANAs between cancer and COPD patients. According to previous reports, age and gender differences can result in different manifestations of ANAs test results. Therefore, our study designed an inter-group balance between genders and ages, and made a comparison between groups after age re-stratification, and discuss the possibility of ANAs to be clinical cancer markers as well. 


\section{Methods}

This case-control study was performed from May 2010 to August 2014 in the Respiratory Medical Department, Qingdao, China. During the study period, lung cancer patients diagnosed by pathology from the respiratory medical department and thoracic surgery department at Qingdao, China, were approached for enrollment in this research. Data were extracted from the records of all patients with mild to very severe COPD and normal control individuals were registered from the respiratory medical department from May 2011 to August 2013.

a. We obtained the final diagnosis of lung cancer by the following methods: Sputum shedding cell examination $(2$ cases), fiberoptic bronchoscopy with bronchial biopsy (32 cases), percutaneous lung biopsy (14 cases), percutaneous pleural biopsy (23 cases), examination for cancer cell in pleural effusion (12 cases), Lymph node biopsy(2 cases), thoracoscopic lung resection(1 cases)?pulmonary lobectomy(4 cases). Specimens were evaluated by three pathologists who had no knowledge of patients' clinicopathologic factors and outcomes. The pathological TNM grading of lung cancer patients was conducted according to the 7th edition NSCLC lymph node metastasis (TNM) clinical staging criteria of the International Lung Cancer Research Association.

b. The diagnosis of COPD was based on ATS/European Respiratory Society (ERS) recommendations, and the severity of COPD was classified according to ATS/European Respiratory Society (ERS) 2005 guidelines. Lung Function Forced spirometry (before and after bronchodilation) [6] measurements in accordance with international guidelines. The reference values were those of the Chinese population [7].

c. The inclusion criteria of COPD were as follows:

(i) Meeting the diagnostic criteria of COPD and no evidence of lung cancer

(ii) No changes in COPD medication dose and symptoms during the first 4 week and

(iii) Age and gender distribution were matched with lung cancer patient

d. The inclusion criteria of normal control group were as follows:

(i) None of the following tests are abnormal: all participants in our study underwent a comprehensive physical examination, and selected laboratory tests (urinalysis, total blood count, erythrocyte sedimentation rate (ESR), blood urea nitrogen (BUN), complement levels $(\mathrm{C} 3, \mathrm{C} 4, \mathrm{C})$, creatine kinase muscle enzyme levels, liver enzymes, and serum creatinine), abdominal organs B-mode ultrasonography, EKG, lung computerized tomography (CT), lung function test

(ii) The patients have no clinical signs or previous medical history

(iii) Age and gender distribution were matched with lung cancer patients

e. The exclusion criteria were: each patient in every group was not allowed to have the following conditions

(i) Other malignant tumors

(ii) Evidence of rheumatic disease, mycobacterial infection, damage of liver or/and kidney function, serious cardiovascular or/ and cerebral vessels system diseases, cardiac arrhythmias disorders, hematological system diseases, environmental exposures, digestive apparatus diseases, diabetes mellitus; mental disease, and other serious infectious diseases

(iii) Pregnancy or lactation women.

\section{Groups Assignment}

A total of 90 cases of lung cancer, 68 cases of COPD and 30 cases of health control people were enrolled in this study. The subjects in each group were divided into two subgroups according to age: under 70 and over 70. Based on the age and gender distribution of patients in lung cancer group and the age subgroups, the age and gender distribution of COPD patients and normal control group were calculated, and patients and subjects were selected according to this requirement.

\section{Measures and Clinical Data}

All selected patients underwent demographic and clinical data collection, collecting venous blood before PSG after a minimum of $8 \mathrm{~h}$ of fasting. Information on thorough medical history, age, gender, medication history and prescription, comorbidities were recorded. Patients in the COPD group were examined for pulmonary function. All measurements were performed by an experienced technician who was not aware of the protocol on the morning of the visit.

\section{Immunofluorescence (IFT)}

After fasting for at least 8 hours, venous blood was collected before PSG and injected into the coagulation tubes immediately, and centrifuged for $10 \mathrm{~min}$, speed $3000 \mathrm{RPM}$ at 20 degrees centigrade. According to the manufacturer's recommendations for the detection of antinuclear reagents by IFT, commercially available kits (EUROIMMUN, Medizinische labordiagnostika AG, Lubeck, Germany) were used. In short, frozen sections of unfixed HEp-2 cells were incubated with serum samples at room temperature for 
30 minutes. After washing with phosphate buffered saline / tween ( $0.2 \%$ PBS tween), sections were exposed to FITC labeled Goat anti human IgG for 30 minutes. Immunofluorescence was observed by two independent researchers using Zeiss immunofluorescence microscope. ANA initial serum dilution was 1/ 10; all samples were diluted to the end of IFT residue. In the negative control group, the slides were incubated with single buffer solution and with FITC labeled antibody respectively. High titer serum or positive serum was used as positive control.

\section{Immunoblot}

Immunoblot was used for specific ANA reactivities---Antiextractable nuclear antibody (ENAs) following the manufacturer's instructions (ANA-Proile3, EUROIMMUN, Germany). ENA results included the following antigens: dsDNA, ribosomal P-protein, histone, CENP-B, JO-1, SCL-70, Ro52, SSA, SSB, Sm, nRNP, PCNA, nucleosome, and M2, obtained by EUROlineScan software (EUROIMMUN, Germany). The illustrations of our tests are shown in Figure 1.

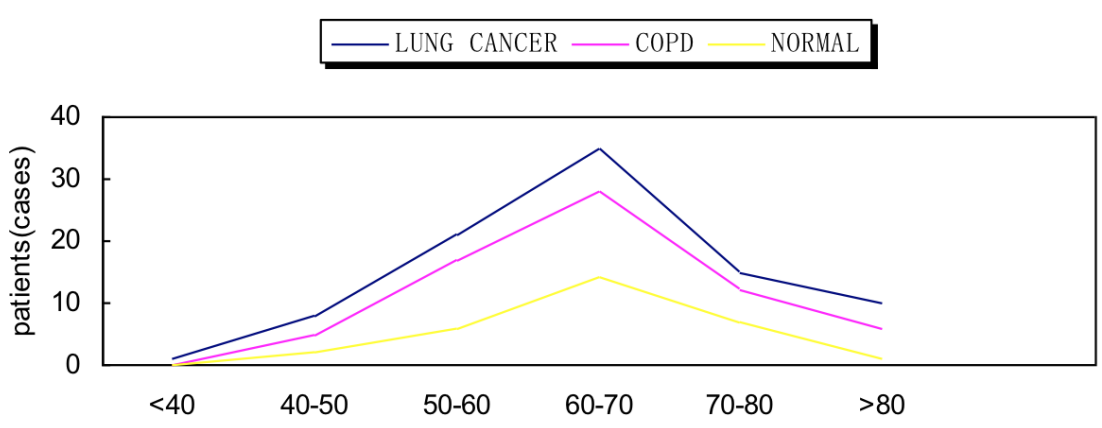

Figure 1: The Characters of age distribution of participating people in different groups

\section{Pulmonary Function Tests}

Including forced vital capacity (FVC, Forced expiratory volume in one second (FEV1), FEVl/FVC\% flow-volume curve, bronchial dilation test were tested based on the American Thoracic Society guidelines, adopting an Germany Jaeger advanced modular spirometer.

\section{Statistical Analysis}

The matching distributions of age and sex between the groups were estimated by calculation. All statistical analyses were performed using SPSS software package (version 17.0; SPSS, Chicago, Illinois, USA). Continuous variables between groups were compared using independent sample $\mathrm{T}$ test. Pearson chi-square analysis and Fisher exact test were used to compare the discrete variables between the two groups. When $\mathrm{P}<0.05$, the difference was considered statistically significant.

\section{Results}

\section{Subjects characteristics}

A total of 90 bronchogenic carcinoma patients were included in this research. The average age was $64.99 \pm 1.18$ years, 66 male patients accounted for $73.33 \%$ of the study population, and 65 , $72.22 \%$, people under the age of 70 . The number of patients with Pathologic TNM stage I, II, IIIA, IIIB and IV lung cancer were 14 (15.56\%), 8 (8.89\%), 10 (11.11\%), 10 (11.11\%) and 48 (53.33\%), respectively. TNM pathological types of lung cancer patients are listed in Table 1.
Table 1: The age and sex distribution characteristics in different group.

\begin{tabular}{|c|c|c|c|}
\hline Variables & $\begin{array}{c}\text { Lung cancer } \\
\text { group }\end{array}$ & COPD group & $\begin{array}{l}\text { Normal } \\
\text { group }\end{array}$ \\
\hline No. of cases & 90 & 68 & 30 \\
\hline \multicolumn{4}{|c|}{ Gender* } \\
\hline Male & 66 & 50 & 22 \\
\hline Female & 24 & 18 & 8 \\
\hline \multicolumn{4}{|c|}{ Age* $^{*}$} \\
\hline$<70$ years & 65 & 50 & 22 \\
\hline$\geq 70$ years & 25 & 18 & 8 \\
\hline Average(years) ${ }^{* *}$ & $64.99 \pm 1.18$ & $63.91 \pm 1.24$ & $65.10 \pm 1.71$ \\
\hline
\end{tabular}

${ }^{*}$ Chi-squared test: Difference were no significant when the ratio of different gender in different group: Lung cancer group compared with COPD group, $\mathrm{P}=0.978$; Lung cancer group compared with normal group, $\mathrm{P}=1.000$; COPD group compared with normal group, $\mathrm{P}=0.984$.

Difference were no significant when the ratio of different age in different group: Lung cancer group compared with COPD group, $\mathrm{P}=0.855$; Lung cancer group compared with normal group, $\mathrm{P}=0.855$; COPD group compared with normal group, $\mathrm{P}=0.984$

${ }^{* *}$ t-test: Data was presented as the mean \pm SEM. Lung cancer group compared with COPD group, $\mathrm{P}=0.536$; Lung cancer group compared with normal group, $\mathrm{P}=0.961$; COPD group compared with normal group, $\mathrm{P}=0.58$.

The average age of 68 COPD patients was $63.91 \pm 1.24$ years, including $50,73.52 \%$, male patients, of whom 50 were under 70 years accounted for $73.52 \%$ of the study population.

According to the severity of ATS/ERS disease, 17 patients (25.00\%), 34 patients (50.00\%), 16 patients (23.53\%), and 1 patient 
$(1.47 \%)$ were classified as mild, moderate, severe, and extremely severe. The clinical data for COPD patients classified by ATS/ERS disease severity are presented in Table 2 . A total of 30 people in the healthy control group were selected, with an average age of $65.10 \pm 1.71$ years, males accounting for $73.33 \%$, and males under
70 years old accounting for $73.33 \%$. There were no statistically significant differences in gender and age distribution between the two groups. The age and gender distribution characteristics of the participants are listed in Table 3. The distributions of age characters of participating people in different groups are in Figure 2.

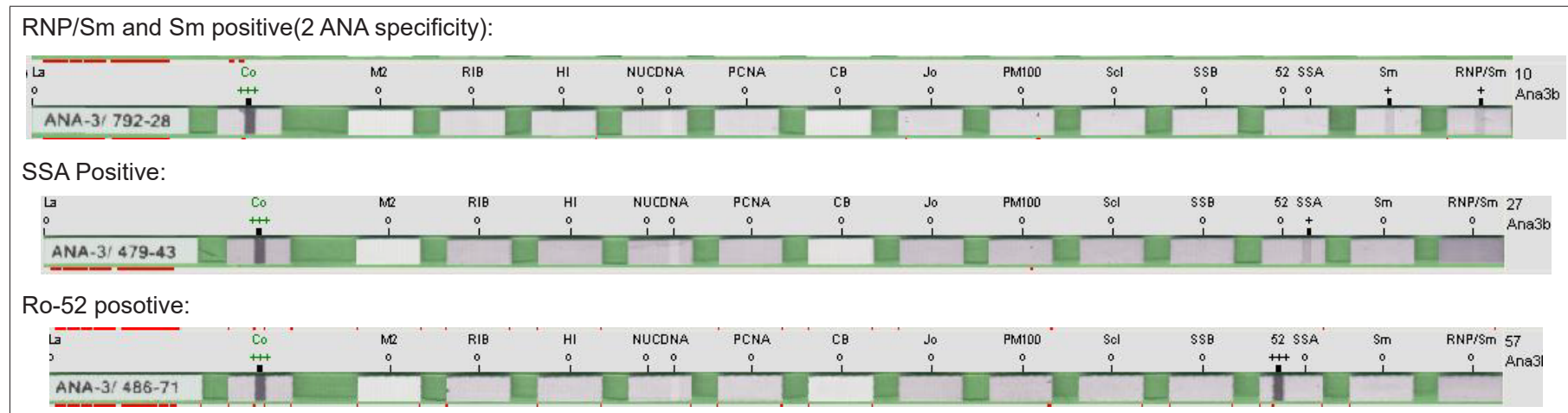

Histones positive:

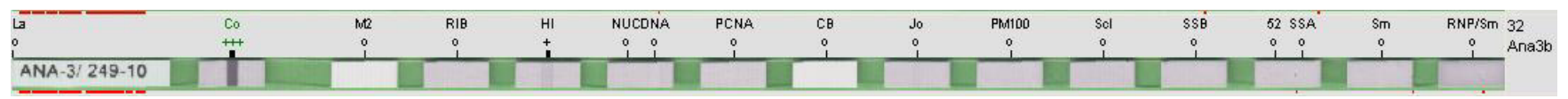

Scl-70 posive:

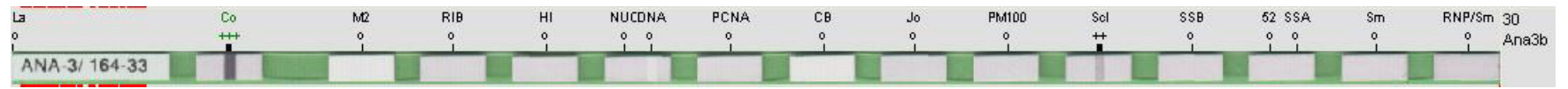

M2 posotive:

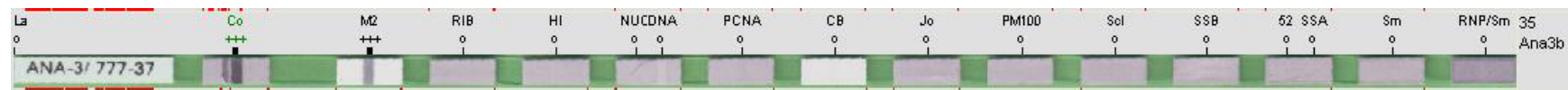

Both Ro-52 and SS-A positive(2 ANA specificity):

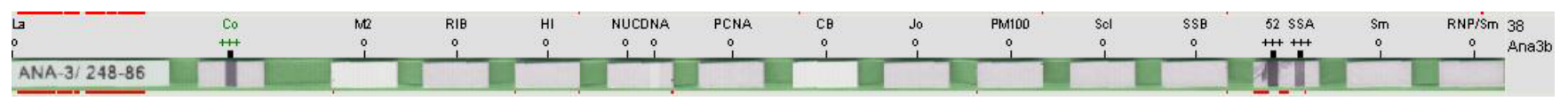

CENP B, Ro-52 and SS-A positive(3 ANA specificity):

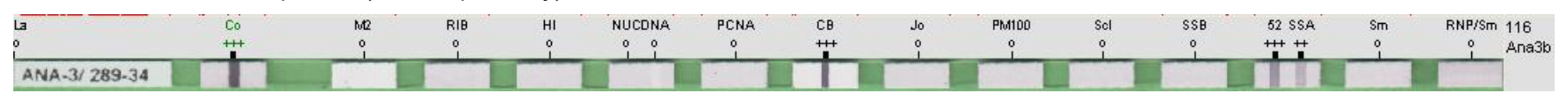

PM-Scl and Ro-52 positive(2 ANA specificity):

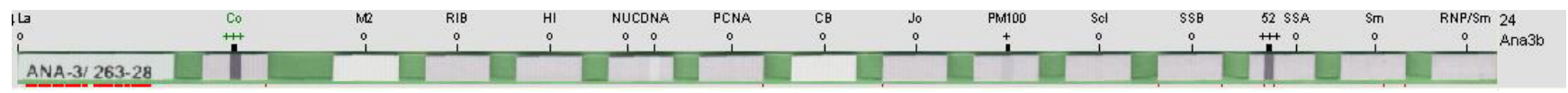

Figure 2: The illustration of our tests:

Table 2: The pathologic TNM classification of the lung patients.

\begin{tabular}{|c|c|c|c|c|c|}
\hline Pathologic TNM Classification & StageI & StageII & Stage III A & Stage III B & Stage IV \\
\hline No. of cases & $14(15.56 \%)$ & $8(8.89 \%)$ & $10(11.11 \%)$ & $10(11.11 \%)$ & $48(53.33 \%)$ \\
\hline
\end{tabular}

Table 3: Clinical data of patients with COPD, by the ATS/ERS classification of disease severity.

\begin{tabular}{|c|c|c|c|c|}
\hline \multicolumn{5}{|c|}{ ATS/ERS Stage of Disease Severity* } \\
\hline & I Mild & II Moderate & III Severe & IV Very severe \\
\hline No. of cases & 17 & 34 & 16 & 1 \\
\hline Males, $\mathrm{n}(\%)$ & $15(88)$ & $24(71)$ & $10(63)$ & $1(100)$ \\
\hline Age, $y$ r, $m$ (SD) & $53.24(4.3)$ & $64.97(4.3)$ & $74.63(7.8)$ & 85 \\
\hline
\end{tabular}

*ATS/ERS stages: I, mild, FEV1/FVC $<0.7$ and FEV1 $\geq 80 \%$ ref; II, moderate FEV1/FVC $<0.7$ and FEV1< $80 \%$ and $\geq 50 \%$ ref; III, severe, FEV1/FVC $<0.7$ and $\mathrm{FEV} 1<50 \%$ and $\geq 30 \%$ ref; IV, very severe, FEV $1<50 \%$ and $\geq 30 \%$ ref. 
The comparison of Antinuclear Autoantibodies Expression between Each Group

The results for each group were illustrated in Table 4. In our study ANAs were much more common in lung cancer patients
$(\mathrm{P}=0.00)$ and $\mathrm{COPD}$ patients $(\mathrm{P}=0.049)$ than in control individuals. Further comparison revealed that circulating ANAs in lung cancer patients were significantly higher than those in COPD patients $(\mathrm{P}=0.004)$.

Table 4: The positive frequency of different Antinuclear Antibodies (ANAs)-patterns in sera from people in subsets of gender with lung cancer, COPD and control.

\begin{tabular}{|c|c|c|c|c|c|c|}
\hline \multirow{2}{*}{ ANAs } & \multicolumn{2}{|c|}{ Lung cancer group $(n=90)$} & \multicolumn{2}{|c|}{ COPD group $(n=68)$} & \multicolumn{2}{|c|}{ Control group $(n=30)$} \\
\hline & Male $(n=66)$ & Female $(n=24)$ & male $(n=50)$ & female $(n=18)$ & male $(n=22)$ & female $(n=8)$ \\
\hline $1: 80$ & 26 & 7 & 4 & 2 & 1 & 1 \\
\hline ANA titer 1:160 & 0 & 0 & 0 & 0 & 0 & 0 \\
\hline $1: 320$ & 0 & 0 & 0 & 0 & 0 & 0 \\
\hline Nrnp/Sm & 1 & 0 & 0 & 0 & 0 & 0 \\
\hline $\mathrm{Sm}$ & 1 & 0 & 0 & 0 & 0 & 0 \\
\hline SS-A & 5 & 4 & 4 & 2 & 0 & 0 \\
\hline Ro-52 & 24 & 4 & 3 & 2 & 0 & 0 \\
\hline SS-B & 0 & 0 & 2 & 0 & 0 & 0 \\
\hline Scl-70 & 1 & 0 & 1 & 1 & 0 & 0 \\
\hline PM-Scl & 1 & 0 & 0 & 0 & 0 & 0 \\
\hline Jo-1 & 0 & 0 & 0 & 0 & 0 & 0 \\
\hline CENP B & 0 & 1 & 2 & 0 & 0 & 0 \\
\hline PCNA & 0 & 0 & 0 & 0 & 0 & 0 \\
\hline dsDNA & 0 & 0 & 1 & 0 & 0 & 0 \\
\hline Nucleosomes & 0 & 0 & 0 & 0 & 0 & 0 \\
\hline Histones & 2 & 1 & 0 & 0 & 0 & 0 \\
\hline Rib.P-protein & 0 & 0 & 2 & 0 & 0 & 0 \\
\hline M2 & 1 & 1 & 0 & 1 & 0 & 0 \\
\hline Positive (\%) & $34(51.5)$ & $10(41.7)$ & $12(24)$ & $6(33.3)$ & $1(4.5)$ & $1(12.5)$ \\
\hline $\mathrm{P}^{*}$ value & \multicolumn{2}{|c|}{0.408} & \multicolumn{2}{|c|}{0.442} & & \\
\hline Positive,total (\%) & \multicolumn{2}{|c|}{$44(48.9)$} & \multicolumn{2}{|c|}{$18(26.5)$} & \multicolumn{2}{|c|}{$2(6.6)$} \\
\hline $\mathrm{P}^{* *}$ value & \multicolumn{6}{|c|}{0.004} \\
\hline
\end{tabular}

The comparison of ANAs positive ratio in different group: Lung cancer group compared with COPD group, $\mathrm{P}=0.004$; Lung cancer group compared with normal group, $\mathrm{P}=0.00$; COPD group compared with control group, $\mathrm{P}=0.049$.

Expression of Antinuclear Autoantibodies in Different Genders

34 ANA positive results were observed in 66 male lung cancer patients (34 out of 66 (51.52\%)), 10 ANA positive results were detected in 24 female lung cancer patients (10 out of 24 (41.67\%)), the difference was not statistically significant $(\mathrm{P}=0.408)$. A higher frequency of elevated positive results was observed in female COPD patients ( 6 out of $18(33.33 \%)$ ) than the male group (12 out of 50 $(24.00 \%))$, the differences were not statistically significant, too ( $\mathrm{p}$ $=0.442$ ) (Table 4).

\section{Expression of antinuclear autoantibodies in different ages}

In lung cancer group, ANA titers were elevated in 31 patients under 70 years of age and one or more ANA specificity out of 65 patients (47.69\%). The increase in ANA titers and one or more ANA specificity were detected in $13(52.00 \%)$ of 25 lung cancer patients aged over 70 years. ANA titer was elevated in 12 patients under 70 years of age in the COPD group, and one or more ANA specificity out of 50 patients (24.00\%). Increased ANA titer and one or more ANA specificities were detected in 6 (33.33\%) of 18 COPD patients over age 70 . No correlation was found between age and ANA positive results in either lung cancer group or COPD group .

\section{Most common types of antinuclear autoantibodies ex- pression}

The most commonly elevated ANA titers observed were ANA titer of 1:80 (33 out of 44 lung cancer ANA-positive and 6 out of 18 COPD ANA-positive). Among the various ANA antibody subtypes, anti-Ro-52 and anti- SSA were the most common. A total of $63.6 \%$ 
of the lung cancer ANA-positive samples available for immunoblot analysis (33 out of 44 ANA-positive) revealed anti-Ro-52, and $20.5 \%$ of the lung cancer ANA-positive samples ( 9 out of 44 ANApositive) were anti-SSA. The prevalence of these autoantibodies in COPD patients showed that 5 of the 18 (27.8\%) ANA-positive patients were anti-Ro-52 antibody-positive, and anti-SSA antibody was present in 6 of 18 (33.3\%) ANA positive patients.

\section{Discussion}

Studies have shown that lung cancer is one of the leading causes of death worldwide. It is a non immunogenic cancer that is resistant to immunomonitoring [8]. Circulating autoantibodies appear more frequently in cancer patients than in non cancer patients [9]. The incidence of ANAs in various types of cancer in different age groups of both sexes had been reported [10]. Our results showed that the ANAs positive rate of lung cancer patients was higher than that of the general population, which was consistent with previous studies.

Chronic obstructive pulmonary disease (COPD) is now the third leading cause of death in the world [11]. And COPD has other important manifestations beyond the lungs, known as systemic effects. These factors include unwilled weight loss, skeletal muscle dysfunction, and an increased risk of cardiovascular disease, osteoporosis, and depression. Low-level chronic systemic inflammation is one of the potential mechanisms of these systemic effects [12]. COPD is a polymerous disease. Autoimmunity may responsible for the pathogenesis of COPD [13]. Lung carcinoma is the most common concurrent comorbidities which COPD patients frequently suffer from, [11] the presence of COPD and emphysema is related to the increased risk factor of lung carcinoma [14].

ANAs react with various nuclear autoantigens. Previous studies have shown that although ANA positive is highly sensitive to certain rheumatic diseases, the presence of ANAs is non-specific and can be involved in a number of non-rheumatic factors, including malignancies, environmental exposure, drugs and infections [15]. They constitute a group of heterogeneous autoantibodies. [16] There are multiple case reports of ANAs in patients with malignancies [16]. The work of Fernandez Madrid F etc showed autoimmunity is a significant feature of squamous cell carcinoma, and suggested that the molecular characteristics of nuclear antigens recognized by ANAs may help to find valuable markers to distinguish LSCC from HNSCC [17]. The group of Nunez B revealed that abnormal titers of circulating ANA occurred in between a quarter and a third of clinically stable COPD patients [18]. Some authors found COPD is a heterogeneous disease and associated with a higher prevalence of ANAs [19].

However, it has been reported that aging is related to autoimmunity, and the positive rate of ANA was higher in the elder $[20,21]$ this trend was not apparent in lung cancer patients in our study when we observed the individuals under 70 compared with over 70 years; Despite some studies suggest ANAs are increased prevalence in women than men [22] Our results show that gender are not strongly associated with ANAs production in lung cancer samples. ANAs positivity was more prevalent in women and over 70 years age patients with COPD but their limited number (only 18 ANA-positive results) restricts the generalizability of this observation.

Although anti-Ro/SSA antibodies are mainly detected in SLE and SS patients, they are sometimes found in other systemic autoimmune diseases [23,24] Ben-Chetrit et al. first discovered a $52 \mathrm{kDa}$ protein Ro52 in Ro antigen in 1988 [25] and the cDNA of human Ro52 was cloned three years later [26,27] In humans, the size of the Ro52 gene is $8.8 \mathrm{~KB}$ which stand on chromosome [11].

Ro52 is an interferon(IFN)-inducible protein [28] which is also induced by type I IFN-induced viral infection or toll-like receptor (TLR) binding [29]. Studies have shown that Ro52 is a negative regulator of proinflammatory cytokine production. And anti-Ro antibodies were frequently found to be involved in SLE, [30] SS/ SLE overlap syndrome, SCLE, and Neonatal lupus erythematosus (NLE) [31] NLE is a passively conveyed autoimmune disease that occurs in mothers of some infants with anti-Ro and / or anti-La antibodies [32] whose most common complication is congenital complete heart block (CCHB), which occurs in $0-5 \%$ of such pregnancies [33]. Anti-ro52 antibody is highly specific in primary biliary cirrhosis, an autoimmune liver disease [34] Although the causative role of autoantibodies in autoimmune diseases is not yet clear, it has been hypothesized that anti-Ro antibody might play a direct role in tissue damage. Anti-ro52 antibody may has pathological effects by directly damaging tissues and inhibiting the activity of Ro52 antigens. Our results revealed in lung cancer patients a higher frequency of anti-Ro-52, followed by anti-SSA. Our study has limitations, we could not assess associations with specific autoimmune diseases or other diseases, and the small sample sizes of some subgroups may limit our ability to detect differences in ANA prevalence on certain factors.

In summary, our results show that a higher frequency of elevated positive results of ANAs was observed in COPD than in normal people, however, the positive rate of circulating ANAs in lung cancer patients was significantly higher than in COPD patients. Further study will help to understand the pathogenesis of COPD and lung cancer and the relationship between the two kind of diseases. The development of autoantibodies is the result of the destruction of human immune balance, [35] and we believe that the main role of the intratumor immune response can promote our understanding of tumor evolution and has important implications in clinical practice [36].

\section{Conflict of Interest}

None. 


\section{References}

1. M Abu Shakra, D Buskila, M Ehrenfeld, K Conrad, Y Shoenfeld (2019) Cancer and autoimmunity: autoimmune and rheumatic features in patients with malignancies. Ann Rheum Dis 60(5): 433-441.

2. R Nisihara, MCC Machoski, A Neppel, CA Maestri, I Messias-Reason, Et al (2018) Anti-nuclear antibodies in patients with breast cancer. Clin Exp Immunol 193(2): 178-182.

3. HPJ Bonarius, CA Brandsma, HAM Kerstjens, JA Koerts, M Kerkhof, et al. (2011) Antinuclear autoantibodies are more prevalent in COPD in association with low body mass index but not with smoking history. Thorax 66(2) :101-107.

4. Belén Núñez, Jaume Sauleda, Josep Maria Antó, Maria Rosa Julià, Mauricio Orozco, et al. (2011) Anti-Tissue Antibodies Are Related to Lung Function in Chronic Obstructive Pulmonary Disease. Am J Respir Crit Care Med 183(8): 1025-1031.

5. Houghton AM (2013) Mechanistic links Between COPD and lung cancer. Nat Rev Cancer 13(4): 233-245.

6. Miller MR, Hankinson J, Brusasco V, Burgos F, Casaburi R, et al. (2005) Standardisation of spirometry. Eur Respir J 26(2): 319-338.

7. Rong-ya Zhao, Lei Zhu, Li Li, Qi Chen, Yan-jie Yang (2011) The applicability of the 1988 version of the prediction equations for adult normal lung function in Shanghai. Zhonghua Jie He He Hu Xi Za Zhi. 34(8): 586-589.

8. Pinto A, Morello S, Sorrentino R (2011) lung cancer and Toll-like receptors. Cancer Immunol Immunother 6(9): 1211-1220.

9. Heegaard NH, West-Norager M, Tanassi JT, Houen G, Nedergaard L, et al. (2012) Circulating antinuclear autoantibodies in patients with pelvic masses are associated with malignancy and decreased survival. PLoS One 7(2): e30997.

10. Imran A, Neelam F, Tariq M (2003) Incidence of circulating antinuclear autoantibodies in cancer patients. Indian J Med Sci 57(3) :113-116.

11. Divo M, Cote C, de Torres JP, Ciro Casanova, Jose M Marin, et al. (2012) Comorbidities and risk of mortality in patients with chronic obstructive pulmonary disease. Am. J. Respir. Crit. Care Med 186(2): 155-161.

12. Agusti A, Soriano JB (2008) COPD as a systemic disease. COPD 5(2): 133138.

13. Nunez B, Sauleda J, Anto JM, Julia MR, Orozco M, et al. (2011) Antitissue autoantibodies are related to lung function in chronic obstructive pulmonary disease. Am J Respir Crit Care Med 183(8): 1025-1031.

14. Gullon JA, Suarez I, Medina A, Rubinos G, Fernandez R, et al. (2011) Role of emphysema and airway obstruction in prognosis of lung cancer. Lung Cancer 71(2): 182-185.

15. Volkmann ER, Taylor M, Ben-Artzi A (2012) Using the antinuclear antibody test to diagnose rheumatic diseases: when does a positive test warrant further investigation? South Med J 105(2): 100-104.

16. MacIntyre N, Crapo RO, Viegi G, Johnson DC, van der Grinten CP, et al. (2005) Standardisation of the single-breath determination of carbon monoxideuptake in the lung. Eur Respir J 26(4): 720-735.

17. Park KJ, Bergin CJ, Clausen JL (1999) Quantitation of emphysema with threedimensionalCT densitometry: comparison with twodimensionalanalysis, visual emphysema scores, and pulmonary function testresults. Radiology 211(2): 541-547.

18. Belén Núñez, Jaume Sauleda, Josep Maria Antó, Maria Rosa Julià, Mauricio Orozco, et al. (2011) Anti-tissue antibodies are related to lung function in chronic obstructive pulmonary disease. Am J Respir Crit Care Med 183(8): 1025-1031.

19. HPJ Bonarius, CA Brandsma, HAM Kerstjens, JA Koerts, M Kerkhof, E Nizankowska-Mogilnicka, et al. (2011) Antinuclear autoantibodies are more prevalent in COPD in association with low body mass index but not with smoking history. Thorax 66(2) :101-107.
20. Candore G, Grimaldi MP, Listi F, Ferlazzo V, Colonna-Romano G, et al. (2002) Prevalence of non organ-specific autoantibodies in healthy centenarians. Arch Gerontol Geriatr Suppl 35:75-80.

21. Nilsson BO, Skogh T, Ernerudh J, Johansson B, Lofgren S, et al. (2006) Antinuclear antibodies in the oldest-old women and men. J Autoimmun 27(4): 281-288.

22. Minoru Satoh, Edward KL Chan, Lindsey A Ho, Kathryn M Rose, Christine G Parks, et al. (2012) Prevalence and Sociodemographic Correlates of Antinuclear Antibodies in the United States. Arthritis Rheum 64(7): 2319-2327.

23. J Schulte-Pelkum, M Fritzler, M Mahler (2009) Latest update on the Ro/ SS-a autoantibody system. Autoimmun Rev 8(7): 632-637.

24. P Ghillani, C André, C Toly, A M Rouquette, D Bengoufa, et al. (2011) Clinical significance of anti-Ro52 (TRIM21) antibodies non-associated with anti-SSA $60 \mathrm{kDa}$ antibodies: results of a multicentric study. Autoimmun Rev 10(9): 509-513.

25. E Ben-Chetrit, EKL Chan, KF Sullivan, EM Tan (1988) A 52-kD protein is a novel component of the SS-A/Ro antigenic particle. J Exp Med 167(5): $1560-1571$.

26. EKL Chan, JC Hamel, JP Buyon, EM Tan (1991) Molecular definition and sequence motifs of the 52-kDcomponent of human SS-A/Ro autoantigen. J Clin Invest 87(1): 68-76.

27. K Itoh, Y Itoh, MB Frank (1991) Protein heterogeneityin the human Ro/ SSA ribonucleoproteins. The 52- and $60-\mathrm{kD}$ Ro/SSA autoantigens are encoded by separate genes.J Clin Invest 87(1): 177-186.

28. Ryusuke Yoshimi, Yoshiaki Ishigatsubo, Keiko Ozato (2012) Autoantigen TRIM21/Ro52 as a possible target for treatment of systemic lupus erythematosus. Int J Rheumatol 718237.

29. Ricardo Rajsbaum, Jonathan P Stoye, Anne O'Garra (2008) Type I interferon-dependent and -independent expression of tripartite motifproteins in immune cells.Eur J Immunol 38(3): 619-630.

30. TT Provost, FC Arnett Jr, M Reichlin (1983) HomozygousC2 deficiency, lupus erythematosus, and anti-Ro (SSA)antibodies. Arthritis Rheum 26(10): 1279-1282.

31. JP Buyon , RJ Winchester, SG Slade, F Arnett, J Copel, et al. (1993) Identification of mothers at risk for congenital heart block and other neonatal lupus syndromes in their children: comparison of enzymelinked immunosorbent assay and immunoblot for measurement of antiSS-A/Ro and anti- SS-B/La antibodies. Arthritis Rheum 36(9): 12631273.

32. Jill P Buyon, Robert M Clancy, Deborah M Friedman (2009) Cardiac manifestations of neonatal lupus erythematosus: guidelines to management, integrating clues from the bench and bedside. Nat Clin Pract Rheumatol 5(3): 139-148.

33. A Brucato, M Frassi, F Franceschini, R Cimaz, D Faden, et al. (2001) Risk of congenital complete heart block in newborns of mothers with anti-Ro/SSA antibodies detected by counterimmuno- electrophoresis: a prospective study of 100 women. Arthritis Rheum 44(8): 1832-1835.

34. A Granito, P Muratori, L Muratori, G Pappas, F Cassani, et al. (2007) Antibodies to SS-A/Ro-52kD and centromere in autoimmune liver disease: a clue to diagnosis and prognosis of primary biliary cirrhosis. Aliment Pharmacol Ther 26(6): 831-838.

35. Alexandru Vlagea, Sandra Falagan, Gerardo Gutiérrez-Gutiérrez, Juan Moreno-Rubio, María Merino, et al. (2018) Antinuclear antibodies and cancer: A literature review. Crit Rev Oncol Hematol 127: 42-49.

36. Bindea G, Mlecnik B, Fridman WH, Pages F, Galon J (2010) Natural immunity to cancer in humans. Curr Opin Immunol 22(2) : 215-222. 